\title{
La Ingeniería \\ de Sistemas en el sector educativo oficial en Colombia
}

Systems engineering in the public education sector in Colombia

\section{Juan Habib Bendeck Soto' \\ jhbendeck@gmail.com \\ Orcid: https://orcid.org/0000-0002-9180-4623}

https://doi.org/10.22209/rhs.v6n2a05

Recibido: julio 23 de 2018.

Aceptado: noviembre 27 de 2018.

\section{Resumen}

El presente artículo tiene como propósito mostrar una reflexión general sobre la Ingeniería de Sistemas como profesión y la perspectiva que maneja el sector educativo público u oficial en Colombia en la actualidad, frente al estudiar o ejercer esta profesión en el campo laboral colombiano. Se analizan diversos aspectos sociales y académicos que influyen en la falta de interés por parte de los estudiantes del sector oficial, teniendo en cuenta que la industria de las Tecnologías de Información y Comunicación (TIC) del país carece de muchos profesionales para cubrir las necesidades actuales del sector empresarial y académico. Por esto, se pretende dar a conocer la importancia de esta industria y se busca una mejor promoción de esta carrera para los sectores sociales inclusivos, en los cuales hace falta una buena cantidad de estudiantes con una formación académica competente para el sector del software.
Palabras clave: Colombia; Educación superior; Inclusión social; Industria de software; Ingeniería de Sistemas; Sociedad.

\section{Abstract}

This paper aims to provide a general reflection on systems engineering as a profession as well as on the current perspective prevalent in the Colombian public educational sector on this discipline both as a degree program and as a profession in the country's labor market. Various social and academic aspects are analyzed that impact the lack of interest on the part of students in the public sector, taking into account that the information and communication technology (ICT) industry in the country lacks a large number of professionals who can meet the current needs of the enterprise and the academic sector. Therefore, this paper aims to raise awareness of the importance of this industry as well as to better promote this degree program among inclusive social sectors, which require a good number of students with a highly qualified academic training for the software sector.

Keywords: Colombia; higher education; social inclusion; software industry; systems engineering; society. 


\section{Introducción}

\section{$\mathrm{I}$}

a Ingeniería de Sistemas en Colombia ha sido una carrera con proyección futurista en cuanto al manejo de tecnologías y a la capacidad de administración que tienen los profesionales que egresan de esta carrera para el desarrollo de soluciones informáticas en todo tipo de industrias.

Y es que, en una carrera donde se están creando aplicaciones nuevas con utilidad para pequeñas, medianas o grandes empresas, en la que se implementan sitios en internet con funcionalidades altamente complejas o la capacidad de conectar equipos de cómputo para crear redes corporativas, se ha empezado a ver como el público recién egresado de las instituciones educativas (sean privadas u oficiales) o distintos profesionales de otros gremios denigren constantemente de las bondades y las facilidades que otorga esta profesión, colocándola como una profesión laboralmente «en vía de extinción».

Esta profesión actualmente en Colombia se ve azotada por la falta de interés y el bajo nivel de ingreso de los estudiantes a las distintas universidades, debido a múltiples paradigmas errados que maneja la sociedad con respecto al concepto de ingeniero de sistemas, los cuales se explicarán a lo largo del desarrollo de este artículo de reflexión, tomando como referencia la población perteneciente al sector oficial, la cual se preocupa únicamente por elegir carreras enfocadas en la formación para el trabajo y el desarrollo humano.

\section{La Ingeniería de Sistemas:}

\section{del amanecer al ocaso}

Remontando hasta veinte años atrás, la Ingeniería de Sistemas en Colombia se mostraba como «la profesión del futuro», la carrera que muchos estudiantes, cuya afinidad con la tecnología o con los computadores era muy fuerte, querían estudiar; y no era para menos, ya que esta carrera prometía garantías económicas estables, una gran proyección laboral con renombre incluido para manejar una empresa o generar grandes soluciones acordes con las necesidades de las pequeñas, medianas y grandes empresas en términos del manejo de información, la resolución óptima y eficiente de las tecnologías de la información, y la oferta de servicios de seguridad informática para la protección de datos de alto valor.

En el mundo, esta industria tiene el privilegio de evolucionar a pasos agigantados comparada con los demás sectores de producción, además de su valía en el Producto Interno Bruto (PIB) nacional, dado que genera altas cantidades de dinero en ventas por producción y servicios; hay que remarcar que, en un país como Colombia, actualmente más del $10 \%$ de la población se encuentra desempleada, y es una de las principales problemáticas para el sector del desarrollo de software y TI en el país: la falta de mano de obra para este sector de producción. Además, hay que tener presente que el Gobierno Colombiano ha gestionado diversos programas de capacitación, como «Colombia Aprende», con el objetivo de conseguir mejores prácticas en el uso de TIC para instituciones 
educativas. Dicho programa permite aportar en el desarrollo de contenidos de calidad, en la definición de uso y apropiación de las tecnologías en la educación, y en la consolidación de las comunidades educativas que den solidez a las políticas de cobertura, calidad y eficiencia de la Revolución Educativa a nivel de la educación preescolar, básica, media y superior (Ministerio de Educación Nacional, 2004). Sin embargo, aún se evidencia la falta de promoción de este campo en el sector educativo oficial que pueda generar mayor producción de profesionales desde la educación superior.

Un estudio, realizado por los investigadores antioqueños Edgar Serna M. y Alexei Serna M. (2013), mostró algunos de los factores por los cuales la Ingeniería de Sistemas se encontraba en declive: el hecho de que Colombia no produzca tecnología de mano propia, que la mayoría de las empresas busquen empleados extranjeros expertos en la materia, que la parte salarial se rebaje al nivel de un técnico o tecnólogo, la falta de presencia del ingeniero dentro de la sociedad, y que la oferta y el perfil exigido no concuerden con lo demandado por las empresas, independiente de la institución de la cual el estudiante se graduó. Mediante esto, se puede comprender por qué esta área potencial pasó a tener menos graduados durante los últimos diez años, superada por un amplio margen por las ciencias humanas o administrativas y contables, como ejemplo.

Debido a esto, existe una preocupación por resaltar: las instituciones educativas oficiales (Básica Primaria, Secundaria o Media) y las instituciones de educación superior requieren atraer y formar talento para las necesidades del sector de las Tecnologías de Información $(\mathrm{TI})$, a partir de los grados primarios en el desarrollo de habilidades esenciales como la lógica matemática, asignatura clave para el mejor desempeño de los estudiantes a lo largo de la carrera. Estos futuros estudiantes con gran talento para la programación o el desarrollo de software han ido desapareciendo o, incluso, su visión de generar nuevos negocios se desvanece por la falta de conocimientos en programación, convirtiéndose en simples esclavos del proceso de reingeniería de lo que ya está desarrollado e implementado, tal como lo afırman Eriksson, Borg, y Borstler (2008), quienes enfatizan que los procesos actuales de ingeniería sufren debido a que se provee poco soporte frente a los altos niveles de re-uso de los procesos que ya se han desarrollado.

Hay que anotar que, según Triantis, Rahmandad y Vaneman (2009), la Ingeniería de Sistemas continúa evolucionando para el desarrollo de procesos de diseño y el ingeniero debe actuar como el integrador entre las diversas disciplinas que comprenden esta carrera, y este es un factor para analizar dentro de esta población. Retomando lo mencionado por Canchala, Serrato y Criollo (2014), esto implica un compromiso por parte de las instituciones educativas de este sector, con el propósito de fomentar la educación basada en igualdad de condiciones para toda la sociedad colombiana, tanto a nivel local como en el extranjero, respetando la normatividad que respalda a estas personas.

Por esto, se dan diversos puntos de vista que exponen que no hay incentivación de este aprendizaje en los colegios públicos, conociendo que estas instituciones, con apoyo del gobierno colombiano o una buena administración, pueden contar con equipos de cómputo adecuados y potenciar así la enseñanza de esta habilidad y de mejorar la capacidad lógica de los estudiantes para la resolución de problemas. Esto a su vez, incluye información errada sobre 
el concepto de Ingeniería de Sistemas, puesto que, al momento de ver que un estudiante es hábil en herramientas ofimáticas (Word, Excel, PowerPoint), se le considera como su futuro perfil profesional sin conocer aún la verdadera razón de ser del ingeniero de sistemas.

Está claro que un país que no promueva un nivel mayor de capacitación en tecnología e innovación se puede considerar marginado del mundo globalizado en el que se vive. A tal punto se extiende esta problemática, que las empresas se están aventurando a contratar jóvenes que aún no han terminado sus estudios, asumiendo el riesgo que implica esto para sus diversas áreas, dependiendo de las capacidades y habilidades que maneje el aprendiz. Esto ha conllevado a que el profesional en Ingeniería de Sistemas se le subvalore por su perfil no ajustable a lo demandado por la industria, la falta de preparación en el pregrado y la baja valoración social que posee a este punto, lo cual sugiere que el crecimiento exponencial en la actual sociedad de conocimiento se apoya más en el valor agregado del sector productivo y está más que claro que para esta población es mucho menor, debido a que los estratos más altos son los que disponen de estas ofertas por su preparación profesional y su visión de la realidad (Organización de Naciones Unidas para la Educación, la Ciencia y la Cultura, 2008).

Miguel Ángel Hernández (2014), en un artículo publicado para el periódico El Tiempo, sostiene que «En Colombia las empresas no han definido la diferencia de perfiles ocupacionales y profesionales entre un técnico en sistemas, un tecnólogo en sistemas y un ingeniero de sistemas, conllevando a que contraten a empleados con un perfil indebido». De igual manera, la oferta y demanda de profesionales altamente calificados, combinándolas con la baja calidad de algunos programas de educación superior deriva en el alto índice de desempleo para esta industria, lo que se refleja en la competitividad que se da entre los profesionales ya graduados con los que recién buscan su oportunidad en el mercado laboral; esta situación muestra el nivel de inconformidad que puedan manejar (graduados y postgraduados) para extender el tiempo de búsqueda y obtener una buena oportunidad laboral, factor que solo incrementará las filas del desempleo en el país (Farné y Vergara, 2008).

\section{Aspectos excluyentes}

\section{del sector educativo público}

\section{frente a la Ingeniería \\ de Sistemas como elección \\ profesional}

Considerando lo anterior, a continuación se describen ciertos aspectos por los que la Ingeniería de Sistemas actualmente continúa en declive en el sector público.

\section{Incompatibilidad de perfiles buscados}

Hoy en día las empresas buscan perfiles profesionales en los que un ingeniero de sistemas debe tener dominio en todo tipo de aplicaciones o herramientas informáticas y enfocarse en desarrollar procesos para organizaciones y fabricar nuevas políticas para generar productos nuevos dentro del sector en el que labore (Hammer y Champy, 1993); esto contrasta con las habilidades que una mayoría pueda aprender y 
desarrollar en la universidad, diferenciado con lo que demandan las nuevas tecnologías emergentes. Según Restrepo (2015), esto es algo grave, ya que la demanda cada vez aumenta y, además, porque ahora las empresas reconocen la diferencia entre un técnico y un tecnólogo. Este factor provoca que todo se contraste con la realidad presente, dado que existen muchas nuevas tecnologías emergentes que no pueden ser cubiertas por los profesionales actuales, porque solo se promueven a unos pocos después de tener en cuenta estudiantes de ingeniería que apuntan a otros sectores (Olver, 2013), o también que los egresados de carreras técnicas o tecnológicas no pueden cubrir estas tecnologías emergentes.

Martínez et al. (2017) resuelven que, en la actualidad, en cuanto a la formación del ingeniero, se está proponiendo la iniciativa de desarrollar un conjunto de capacidades, valores y comportamientos compatibles dentro de los nuevos roles que debe asumir el egresado en Ingeniería de Sistemas, los cuales son propios para este profesional, pero deberán caracterizar a otros ingenieros y a otros profesionales dentro de las ciencias de la computación.

\section{Falta de mercadeo de parte de las instituciones (Educación Básica, Media y Superior)}

La mayoría de universidades del país se concentran en promover carreras relacionadas con la administración, las leyes, las ciencias humanas u otras ingenierías, en lugar de promover la Ingeniería de Sistemas con mayor intensidad en un país que precisa más expertos en TIC y en el desarrollo de nuevas aplicaciones.
En el sector de educación básica y media, los colegios públicos y privados concentran su enfoque pedagógico en otras áreas de conocimiento, tomando el área de Tecnología e Informática como un campo de conocimiento complementario, mas no relevante para la futura formación técnica, tecnológica o profesional del estudiante.

\section{Índices de deserción en las instituciones de educación superior}

Según Lina Velásquez (2016), en Colombia, actualmente existen alrededor de 93 programas de ingeniería, increíblemente más que los ofrecidos por países como Brasil (48) y México (45); sin embargo, el índice de deserción continúa siendo muy alto y el número de egresados no satisface lo exigido actualmente por la industria de TIC del país, debido a que solo mil ingenieros alcanzan a graduarse por año de todas las universidades colombianas.

Rojas (2009) manifiesta que la deserción universitaria va asociada con factores como las presiones económicas, académicas, laborales e incluso psicológicas. Además, causas comunes a este fenómeno son el disgusto frente al programa, el promedio de la materia estaba por debajo del exigido, y la falta de apoyo por parte de la universidad.

Por esta razón, diversos estudios han sido efectuados en distintas universidades del país a modo de artículos o proyectos de investigación, para detectar las principales causas de deserción de este programa, e indagar qué conlleva a los estudiantes a no continuar estudiando esta 
carrera. Casos tales como la Universidad de la Amazonía, Universidad del Quindío, Universidad Católica de Colombia, Universidad Simón Bolívar, entre otras, han realizado proyectos de investigación para detectar estos factores; cifras obtenidas de esta investigación eran significativas dentro del programa, puesto que entre el 12 y el $20 \%$ de los matriculados desertaron, o solo el $15 \%$ de los matriculados al programa terminaron la carrera, o inclusive, por razones no especificadas o indeterminadas por parte de los estudiantes (Romero y Paredes Niz, 2017). También cabe resaltar que, a pesar de que las universidades promuevan estrategias y manejen incentivos para motivar a los estudiantes a permanecer, aún continúan prevaleciendo los índices de deserción (Higuera, 2016).

Estos aspectos son apenas una muestra de lo que se ve en nuestro país. Por ejemplo, desde las instituciones, ¿qué tanto se les motiva a los estudiantes a estudiar este tipo de carreras? Además, la familia también juega un rol importante para la motivación del estudiante, esto junto con la influencia de docentes para fomentar más la importancia de esta industria. ¿Y qué hay de las instituciones educativas? ¿Acaso no alcanzan a dimensionar la cantidad de tecnologías que actualmente se encuentran huérfanas de ingenieros que puedan manejarlas y dominarlas?

Esto nos marca una gran diferencia entre lo que puede acontecer en países americanos o europeos con respecto al nuestro, en donde se le hace un énfasis global y general en el estudio y el desarrollo de las habilidades para estudiar una carrera como la Ingeniería de Sistemas o carreras relacionadas con la industria del software, reconociendo la urgencia y la necesidad que se demanda para el mejor desarrollo del país en el sector internacional; y es que el país, si se propone realizar alianzas estratégicas con empresas pertenecientes a esta industria y juntarlas con el sector educativo, puede potenciar la capacitación de toda la educación en todos los niveles.

\section{Conclusiones}

Luego del análisis previo de estos factores de rechazo y de desconocimiento frente a esta carrera por parte del sector público, cabe reflexionar sobre las medidas que deben tomar tanto el Gobierno colombiano como el sector privado, así como la gestión académica de parte de los colegios oficiales y la mejor educación en las instituciones de educación superior, en el marco de mejorar la promoción y formación de estos profesionales. De acuerdo con lo mencionado, se puede concluir que:

- El Gobierno y las instituciones educativas de básica secundaria deben diseñar e implementar estrategias más agresivas para mejorar la calidad no solo de los estudiantes que apenas se forman, sino también de los que están a punto de graduarse, pensando en la mejor capacitación de las nuevas tecnologías emergentes: sostenibilidad de la información, globalización de conocimiento y, además, la industria del desarrollo de software (desarrollo de aplicaciones móviles, desarrollo de aplicaciones de escritorio y desarrollo de videojuegos, Big Data, entre otras más).

- El concepto de educación inclusiva debe apuntar a responder a la diversidad de necesidades de todos los estudiantes, especialmente en el área de sistemas, que permita generar cambios y modificaciones en los contenidos, aproximaciones, estructuras y 
estrategias, con una visión global y la convicción de que es la responsabilidad del sistema educativo promover esto para todas las personas del sector público.

- Es claro que existe una problemática en la formación en competencias básicas en educación básica y media, lo cual provoca que los estudiantes opten por carreras donde no se incluyan las matemáticas; razón por la cual, programas de ciencias humanas, sociales y administrativas resaltan más para estos estudiantes. Por ende, se debe fomentar más el desarrollo de competencias lógico-matemáticas en estas instituciones si se desea que los futuros ingenieros destaquen en elementos como la innovación y el desarrollo.

\section{Referencias}

Bolivar, A. (2008). Ciudadanía y competencias básicas. Sevilla: Fundación ECOEM.

Canchala, M., Serrato, M., \& Criollo, C. (2014). Diagnóstico sobre inclusión educativa en discapacidad en los programas de pregrado de la Universidad Mariana. Boletín Informativo CEl, 7(2), 92-95.

Eriksson, M., Borg, K., \& Borstler, J. (2008). Use Cases for Systems Engineering - An Approach and Empirical Evaluation. Systems Engineering, 17(1), 39-60.

Farné, S., \& Vergara, C. (2008). Cuaderno 10. Recuperado de Universidad de Externado Colombia: https://bit.ly/2DYOr8F

Hammer, M., \& Champy, J. (1993). Reengineering the Corporation. Nueva York: Harper Collins.

Hernández, M. (10 de febrero de 2014). Industria TIC colombiana necesita muchos más ingenieros. El Tiempo.
Higuera, O. (2017). Deserción estudiantil en Colombia y los programas de Ingeniería de la UPTC Seccional Sogamoso. Ingeniería, Investigación y Desarrollo, 17(1), 70-76. Recuperado de https:// bit.ly/2SgRSuy

Martínez, A., Jímenez, R., \& Hernández, G. (2017). Rol del Ingeniero de Sistemas en Colombia. Boletín Informativo, Universidad Mariana, Bogotá, D.C.

Ministerio de Educación Nacional. (2004). La nueva red del conocimiento. Recuperado de Sitio Web del Ministerio de Educación Nacional: http://www. mineducacion.gov.co/1621/article-87398.html

Olver, R. (24 de junio de 2013). Lack of engineers means Britain III-prepared for future. The Telegraph.

Organización de las Naciones Unidas para la Educación, la Ciencia y la Cultura. (Noviembre de 2008). La educación inclusiva: El camino hacia el futuro. Recuperado de UNESCO: https://bit.ly/2wFOKhd

República de Colombia. (1991). Constitución Política de Colombia. Bogotá, D.C.

Restrepo, P. (2015). La gente no quiere estudiar Ingeniería. (E. Bohórquez, Entrevistador) Colombia: El Espectador.

Rojas, M. (2009). El abandono de los estudios: deserción y decepción de la juventud. Revista Académica de la Facultad de Ciencias Sociales, 4.

Romero, G., \& Paredes, A. (2017). Análisis de la deserción estudiantil en la USB, facultad Ingeniería de Sistemas, con técnicas de minería de datos. Revista I+D en TIC, 4(1), 13-18.

Serna, M., \& Serna, A. (2013). La formación en Ingeniería en Colombia: Una situación que preocupa. Medellín. Recuperado de Universidad.com.co.

Triantis, K., Rahmandad, H., \& Vaneman, W. (2009). Systems Engineering: Have we lost our Competitive Edge? A consideration of the Dynamics of Systems Engineering Projects, (pp. 3-22). Falls Church, Virginia.

Velásquez, L. (15 de Abril de 2016). En Colombia se necesitan 18.000 ingenieros más. El Colombiano. Recuperado de https://bit.ly/1SGscjm 